\title{
Secukinumab
}

\section{Positives Votum des CHMP}

Novartis gab im November bekannt, dass der Ausschuss für Humanarzneimittel (CHMP) eine positive Stellungnahme abgegeben hat, in der er die Zulassung von Secukinumab (vormals AIN457) als systemische Erstlinientherapie von mittelschwerer bis schwerer Plaque-Psoriasis bei Erwachsenen, die für eine systemische Therapie in Frage kommen, empfiehlt.

Die Empfehlung als Erstlinientherapie beinhaltet, dass Secukinumab als Alternative zu anderen systemischen Erstlinien-Therapeutika zur Verfügung steht [4]. Die anderen Biologika zur Behandlung von Psoriasis werden im Gegensatz dazu in Europa als systemische Zweitlinientherapie empfohlen; dies schließt die TumornekrosefaktorBlocker (TNF-Blocker) und Ustekinumab mit ein [5-7].

Secukinumab (300 mg) ist damit der erste Interleukin-17A-(IL-17A-)Inhibitor, der in Europa als Erstlinientherapie zur Behandlung von Psoriasis-Patienten, für die eine systemische Therapie angezeigt ist, empfohlen wird. Das Biologikum Secukinumab hemmt die Wirkung von IL-17A, einem Protein, das in hohen Konzentrationen in der erkrankten Haut nachgewiesen wird [8-13].

Wichtigstes Ziel der Psoriasis-Behandlung ist das Erreichen einer symptomfreien Haut. In klinischen Studien erzielten mindestens $70 \%$ der Patienten unter Secukinumab 300 mg während der ersten 16 Wochen der Behandlung eine symptomfreie (PASI 100- eine $100 \%$ ige Verbesserung der Hautsymptomatik) oder fast symptomfreie Haut (PASI 90- eine 90\%ige Verbesserung der Hautsymptomatik) $[1,14]$.

Die Stellungnahme des CHMP stützte sich auf die positiven Ergebnisse des klinischen Studienprogramms der Phase III zu mittelschwerer bis schwerer Plaque-Psoriasis und folgt damit der einhelligen Empfehlung für eine Zulassung, die das Dermatologic and Ophthalmic Drugs Advisory Committee (DODAC) im Oktober gegenüber der US Food and Drug Administration (FDA) abgegeben hat $[1-3,14]$.

\section{ERASURE und FIXTURE}

Im Rahmen dieser Studien sorgte Secukinumab durchweg für eine sehr hohe Ansprechrate der Haut und es bewies darüber hinaus Überlegenheit gegenüber Etanercept in der Head-to-HeadStudie FIXTURE $[1-3,14]$.

Mindestens $70 \%$ der Patienten erzielten in den Studien FIXTURE und ERASURE unter Secukinumab 300 mg während der ersten 16 Wochen der Behandlung eine symptomfreie Haut (PASI 100) oder fast symptomfreie Haut (PASI 90 ) - 72,4\% und $36,8 \%$ für PASI 90 bzw. $100(p<0,001$ für alle Vergleiche) - dieser Erfolg konnte bei fortgesetzter Behandlung bei der Mehrheit der Patienten bis Woche 52 aufrechterhalten werden [1-3, 14]. In den Studien FIXTURE, ERASURE, FEATURE und JUNCTURE (die von der US-amerikanischen FDA geprüft wurden) bemerkten die Patienten in den Secukinumab-Gruppen deutliche Verbesserungen bereits nach 2 Wochen. Im Durchschnitt wurde ein Rückgang der Symptome um $50 \%$ unter Secukinumab $300 \mathrm{mg}$ bis Woche 3 verzeichnet, unter Etanercept in der FIXTURE-Studie dauerte dies 7 Wochen $(p<0,001$ für alle Vergleiche) $[1-3,14]$. Secukinumab zeichnete sich durch ein günstiges Sicherheitsprofil aus; die Häufigkeit und Schwere von unerwünschten Ereignissen waren in beiden Secukinumab-Behandlungsarmen ( $300 \mathrm{mg}$ und $150 \mathrm{mg}$ ) vergleichbar, auch im Vergleich zu Etanercept zeigt sich kein Unterschied hinsichtlich des Auftretens unerwünschter Ereignisse [1-3, 14].

Die Europäische Kommission prüft die Empfehlungen des CHMP. Die abschließende Entscheidung bezüglich der Zulassung wird in der Regel etwa zwei Monate nach der Stellungnahme des CHMP gefällt und wird für alle Länder der Europäischen Union (EU) und des Europäischen Wirtschaftsraums (EWR) Gültigkeit haben.

\section{Secukinumab und Interleukin-17A}

Secukinumab ist ein vollständig humaner monoklonaler Antikörper, der selektiv IL-17A neutralisiert [8, 9]. IL-17A findet sich in hohen Konzentrationen in den von Psoriasis betroffenen Hautarealen und gilt als bevorzugter Angriffspunkt für in der Erprobung befindliche Therapeutika [8-13].

Derzeit laufen Phase-Illb-Studien zu Psoriasis, wie unter anderem die Headto-head-Studie CLEAR, im Rahmen derer Secukinumab im Vergleich zu Ustekinumab bei mittelschwerer bis schwerer Plaque-Psoriasis untersucht wird; außerdem laufen Studien zu Psoriasis palmoplantaris, Nagelpsoriasis und Psoriasis pustulosa palmoplantaris.

Secukinumab wird daneben in Studien der Entwicklungsphase III zur Behandlung von Psoriasis-Arthritis und Spondylitis ankylosans untersucht; Zulassungsanträge für die Anwendung von Secukinumab bei diesen arthritischen Indikationen sind für 2015 geplant.

hautnah $2015 \cdot 14: 3$

DOI 10.1007/s12326-015-0154-4 Online publiziert: 29. Januar 2015 (C) Springer-Verlag Wien 2015
Quelle:

Pressemitteilung

Novartis Pharma $\mathrm{GmbH}$

Literatur auf Anfrage 